\title{
Damage cluster distributions in numerical concrete at the mesoscale
}

\author{
Okan Yllmaz, ${ }^{1, *}$ Peter Michael Derlet, ${ }^{2}$ and Jean-François Molinari ${ }^{1}$ \\ ${ }^{1}$ Civil Engineering Institute, Materials Science and Engineering Institute, École Polytechnique Fédérale de Lausanne (EPFL), Station 18, \\ CH-1015 Lausanne, Switzerland \\ ${ }^{2}$ Condensed Matter Theory Group, Paul Scherrer Institute, CH-5232 Villigen PSI, Switzerland
}

(Received 11 October 2016; revised manuscript received 8 March 2017; published 26 April 2017)

\begin{abstract}
We investigate the size distribution of damage clusters in concrete under uniaxial tension loading conditions. Using the finite-element method, the concrete is modeled at the mesoscale by a random distribution of elastic spherical aggregates within an elastic mortar paste. The propagation and coalescence of damage zones are then simulated by means of dynamically inserted cohesive elements. Dynamic failure analysis shows that the size distribution of damage clusters follows a power law when a system-spanning cluster is first observed, with an exponent close to that of percolation theory. This is found for a range of selected mesostructural parameters, material defects, and applied strain rates. In all cases, the system-spanning cluster occurs prior to the onset of local decohesion, a regime of crack nucleation and propagation, and eventual material failure. The resulting fully damaged crack surfaces after failure are found to be only weakly correlated with the percolated damage region structures.
\end{abstract}

DOI: 10.1103/PhysRevE.95.043002

\section{INTRODUCTION}

Understanding failure is crucial to the design of optimal materials and structures. Disorder at different length and time scales, either preexisting or evolving during deformation, plays a fundamental role in the physics of material failure. While microscopic disorders can be vacancies, inclusions, dislocations, or grain boundaries, at the macroscale, these spatial inhomogeneities can be viewed as random, spatially varying, local material parameters [1]. In the statistical physics community, failure in disordered materials is generally studied with random models, in which the disorder is treated by assigning random properties to the elements of the model material. There are different classes of models depending on the type and properties of the failure process of interest. These models include, but are not limited to, random fuse models [2-4], central force models [5], elastic beam models [6], fiber bundle models [7-10], molecular dynamics models [11], and finite-element models with progressive damage [12]. For broader reviews of the models mentioned above, refer to Refs. [1,13-15].

Concrete is composed of mortar paste and coarse aggregates at the mesoscale. Hence, its composite structure can be modeled by discretizing the domain using the geometrical information of aggregate particles and assigning different material parameters to the finite elements. The proposed model is a more realistic description of the material since the heterogeneity is now represented explicitly by variations in microstructure at the mesoscopic level. The weakest part of concrete is the interfacial transition zone (ITZ), the region surrounding the aggregates. The gradient of water-cement ratio around the aggregate particles during casting results in a different microstructure of the surrounding hydrated cement paste [16]. The weak zones of ITZ are randomly distributed within the domain following the aggregate size distribution. Damage within the concrete sample initiate from

\footnotetext{
*okan.yilmaz@epfl.ch; jean-francois.molinari@epfl.ch
}

these weak zones and develop following a complex growth and coalescence mechanism under loading, eventually leading to internal crack evolution and material failure.

In this paper, we mainly focus on the statistics of these damage clusters prior to cracking, formed within the dynamic failure response of concrete at the mesoscale, and propose analogies with percolation theory [17] at a structural level. In Sec. II, we describe the geometrical details of the concrete mesostructure, the size and spatial distribution, and placement of the aggregates. The mechanical problem and numerical setup is explained in Sec. III. Section IV presents the cluster statistics, the evolution of maximum cluster size, the number of independent clusters, and their size distribution. The clusters are studied using different mesostructures (characterized by a particular random distribution of fracture parameters), altering the aggregate content and for different sample sizes. For the study of the corresponding mechanical properties several strain rates are considered. During loading, the spatial evolution of regions undergoing damage (plastic deformation) and fracture is studied. It is found that the spatial cluster statistics of these plastic regions is well described by a power-law distribution whose exponent suggests percolation physics is at play. It is further found that percolation of the plastic regions occurs prior to the peak stress in the corresponding stress-strain curve. In Sec. V it is found that the geometry of the eventual fracture surface is only weakly related to the plastic structure at percolation demonstrating that yield and failure are distinct mechanical regimes. It is also shown that the crack localization is lost at higher rates as damage zones are more likely to become free crack surfaces. Avalanche behavior is also discussed highlighting the differences between our model and classical random models.

\section{CONCRETE MESOSTRUCTURE}

Concrete is modeled by coarse aggregates, mortar paste, and the interfacial transition zone between them. The shape of an aggregate depends on its type. Generally, crushed rock aggregates have an angular shape while gravel aggregates are 
(a)

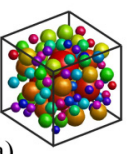

(b)
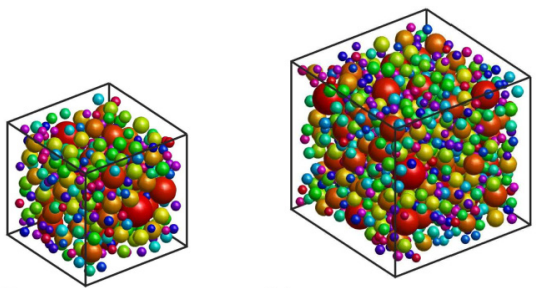

(c)

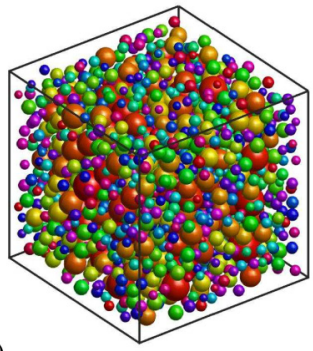

(d)

FIG. 1. Computer-generated mesoscale samples with different simulation box sizes: (a) $L=40 \mathrm{~mm}$, (b) $L=60 \mathrm{~mm}$, (c) $L=80$ $\mathrm{mm}$, (d) $L=100 \mathrm{~mm}$, and (e) $L=120 \mathrm{~mm}$. The packing density is $30 \%$ for all samples.

of a rounded shape [18]. For simplicity, we use spherical aggregates in our models. The aggregate particle size distribution is obtained with a sieving method that uses the Fuller's distribution [19]. It is formulated as

$$
P(d)=100\left(\frac{d}{d_{\max }}\right)^{n},
$$

where $P(d)$ is the total percentage of aggregates that pass a sieve of diameter $d, d_{\max }$ is the maximum aggregate diameter, and $n$ is an exponent, which typically takes values between 0.45 and $0.7 ; n=0.5$ is used for all the models in this work. To generate a particular mesostrucure, we place the coarse aggregates into the sample using a random placement technique named the "take-and-place" method [20]. Here a minimum distance criterion between aggregates is enforced to avoid aggregate overlap. For example, the minimum distance between the centers of the spherical aggregates $i$ and $j$ should be at least $(1+\eta)\left(r_{i}+r_{j}\right)$, where $\eta$ is the minimum distance parameter and $r_{i}$ is the radius of $i$ th aggregate. Analysis (not given here) shows that the distribution exponent $(n)$ and minimum distance parameter $(\eta)$ do not significantly affect the findings of the present work.

For the present work, different realizations of aggregates mesostructures are generated using the above stochastic method. The diameter of the maximum aggregate is $16 \mathrm{~mm}$ while the minimum is taken as $4 \mathrm{~mm}$. A number of different packing densities $(20 \%, 30 \%$, and $40 \%)$ for different systems sizes will be considered. Here the packing density for concrete is defined as the volumetric percentage of the aggregates in the sample. Including sand, realistic packing densities for concrete can go up to $60 \%-70 \%$. However, these values are not currently tractable, and therefore we will consider the regime of coarse aggregates at lower packing densities. Figure 1 displays a range of sample sizes at a packing density of $30 \%$. The samples are cubic, and the largest has a side length of $L=120 \mathrm{~mm}$. Since the maximum aggregate size is smaller than one-sixth of the side length of the reference sample, the computer-generated mesoscale models with $L \geqslant 100 \mathrm{~mm}$ can be considered as representative volumes [21], which means that a calculation using these volumes should yield a value that is representative of the macroscopic properties. The samples with $L=100 \mathrm{~mm}$ will be investigated in detail and considered as our reference system.

\section{NUMERICAL MODEL}

The coarse aggregates and mortar paste are represented with linear elastic continuum finite elements. Using the finiteelement model, we solve the equation of motion

$$
\mathbf{M u ̈}+\mathbf{f}^{\text {int }}=\mathbf{f}^{\text {ext }},
$$

where $\mathbf{M}$ is the mass matrix, $\mathbf{u}$ is the displacement vector, and $\mathbf{f}^{\text {int }}$ and $\mathbf{f}^{\text {ext }}$ are the internal and external force vectors respectively. This equation is solved at every time step using an explicit time integration scheme and the displacement field is updated. For further details regarding the implementation, see Ref. [22]. Crack initiation and propagation is modeled via cohesive elements, which are inserted at the interelement boundaries when the local stress exceeds a critical stress threshold. This criterion is a material property and depends on the material of the elements neighboring the cohesive surface. Such cohesive elements are also used to model the ITZ.

The samples are loaded in uniaxial tension under displacement control with an imposed strain rate. A constant velocity of $V_{0}=0.5 \dot{\varepsilon} L$ is applied to the top and bottom boundaries. No velocity is applied in the transversal directions, and free boundary conditions are imposed. Note that the numerical setup exhibits a Poisson effect, which is natural for the material response of concrete. To avoid the stress wave propagation and an early fracture near the boundaries, an initial velocity profile is applied as a function of the vertical position:

$$
V_{y}(y, t=0)=\frac{2 V_{0}}{L} y,
$$

where $y=0$ is the midplane of the sample. The finite-element code used for the study is based on a Newmark explicit time integration scheme, which is conditionally stable when the time step is less than a critical value set by the CourantFriedrichs-Lewy condition [23]:

$$
\Delta t_{\text {crit }}=\alpha \min _{e=1 \ldots N}\left(\frac{l_{e}}{c}\right),
$$

where $c$ is the pressure wave speed of the associated material and $l_{e}$ is the size of element $e$. The smallest value of the calculated time step over all elements is the chosen time step for the simulation. A security coefficient, $\alpha$, is also used $(0.1$ for this study) to ensure stability.

The used material properties for the elastic and cohesive elements are given in Table I. Constant material properties are mainly employed to limit the damage initiation mechanism to the geometrical details of the mesostructure. Random distributions, in which the material parameters are drawn from a chosen distribution, will also be considered. 
TABLE I. Material properties of aggregate particles, mortar paste, and the interfacial transition zone (ITZ) [24].

\begin{tabular}{lccc}
\hline \hline & Aggregate & Mortar & ITZ \\
\hline Young's modulus, $E[\mathrm{GPa}]$ & 75 & 25 & - \\
Poisson's ratio, $v$ & 0.2 & 0.2 & - \\
Density, $\rho\left[\mathrm{kg} / \mathrm{m}^{3}\right]$ & 2700 & 2200 & - \\
Fracture energy, $G_{\mathrm{c}}\left[\mathrm{J} / \mathrm{m}^{2}\right]$ & 60 & 50 & 30 \\
Tensile strength, $f_{\mathrm{ct}}[\mathrm{MPa}]$ & 10 & 4 & 2.4 \\
\hline \hline
\end{tabular}

\section{Cohesive element method}

The cohesive element method is used to model dynamic fracture in concrete. The method is based on the cohesive crack model of Dugdale [25] and Barenblatt [26]. To represent the decohesion we bury all complex debonding processes in a phenomenological, simple, cohesive law relating the traction and opening displacement. This constitutive response is called traction-separation law:

$$
\mathbf{T}=\mathbf{T}(\Psi)
$$

where $\mathbf{T}$ is the traction acting on the separating surfaces and $\boldsymbol{\Psi}$ is the relative opening displacement vector. We use a simple linear irreversible softening law [27,28]. The existing free potential energy $\phi$, is assumed to depend only on one effective scalar displacement $\delta$ of the following form:

$$
\delta=\sqrt{\Psi_{n}^{2}+\beta^{2} \Psi_{s}^{2}},
$$

where $\Psi_{n}$ and $\Psi_{s}$ are the normal and tangential displacement components while $\beta$ is the parameter that couples these two displacements. We choose $\beta=1$. The free potential energy is

$$
\phi(\delta)=\frac{1}{2} f_{\mathrm{ct}} \delta\left(2-\frac{\delta}{\delta_{\mathrm{c}}}\right),
$$

where $f_{\mathrm{ct}}$ is the critical stress associated within the cohesive element and $\delta_{\mathrm{c}}$ is the effective relative displacement value beyond which complete decohesion occurs.

The derivation of the free potential energy with respect to the opening displacement gives the cohesive tractions:

$$
\mathbf{T}=\frac{\partial \phi}{\partial \Psi}=\frac{T}{\delta}\left(\Psi_{n} \mathbf{n}+\beta^{2} \Psi_{s} \mathbf{s}\right),
$$

where $\mathbf{n}$ and $\mathbf{s}$ are the unit vectors in normal and tangential directions. The crack opening condition is denoted with $\delta=$ $\delta_{\max }$ and $\dot{\delta}>0$, and the crack closure and reopening case is stated as $\delta<\delta_{\max }$. Thus, the traction is

$$
T=\left\{\begin{array}{ll}
f_{\mathrm{ct}}\left(1-\frac{\delta}{\delta_{\mathrm{c}}}\right) & \text { for } \delta=\delta_{\max } \text { and } \dot{\delta}>0 \\
\frac{\delta}{\delta_{\max }} T_{\max } & \text { for } \delta<\delta_{\max }
\end{array},\right.
$$

where $\delta_{\max }$ is the maximum value of the achieved effective displacement up to that instant and $T_{\max }$ is the associated traction value for maximum relative displacement.

Damaging of the cohesive element is an irreversible process while the traction-separation law allows recovering energy upon unloading. Damage in a cohesive element is denoted as

$$
D=\min \left(\frac{\delta_{\max }}{\delta_{\mathrm{c}}}, 1\right) \text {. }
$$

The total dissipated fracture energy during the separation process is equal to the total area under the traction-separation curve: $\frac{1}{2} f_{\mathrm{ct}} \delta_{\mathrm{c}}$. When total decohesion occurs two free surfaces are formed at the interelement boundaries.

Cohesive-zone modeling introduces another length scale set by the elastic and fracture properties. This length scale can be expressed for simple cases (mode I loading, homogeneous material) as [29,30]

$$
l_{z}=\frac{E G_{c}}{f_{\mathrm{ct}}^{2}} .
$$

The cohesive length scales for aggregate and mortar are 78.1 $\mathrm{mm}$ and $45 \mathrm{~mm}$, respectively. Since the ITZ is not represented with continuum elements and does not have a defined elastic modulus, its associated cohesive length scale is ill-defined.

The condition $l_{\mathrm{m}}<l_{\mathrm{z}}$ between the length scales has to be satisfied in our simulations where $l_{\mathrm{m}}$ is the biggest element size. Obviously, the simulation-box size is an additional length scale in our simulations, and the interplay of all length scales is crucial for the understanding of the obtained results. The cohesive zone must contain several elements (typically around four [31]) and should be small compared to the sample size for mesh independency. The average element size for the analyses, which is the average side length for a regular tetrahedron element, is $2.1 \mathrm{~mm}$. The most constraining cohesive zone length is around $45 \mathrm{~mm}$ for our simulations, which clearly satisfies the requirement. However, the cohesive zone length is not small compared to our sample size. Unfortunately, bigger mesostructures cannot be simulated due to the computational limitations.

\section{DAMAGE CLUSTERS}

As the reference case, we consider the mesostructure shown in Fig. 1(d) for a strain rate of $\dot{\varepsilon}=1 \mathrm{~s}^{-1}$.

The macroscopic stress-strain response is plotted in Fig. 2. Damage zones initiate early at the prepeak phase. The start of damaging indicates the insertion of the first cohesive element

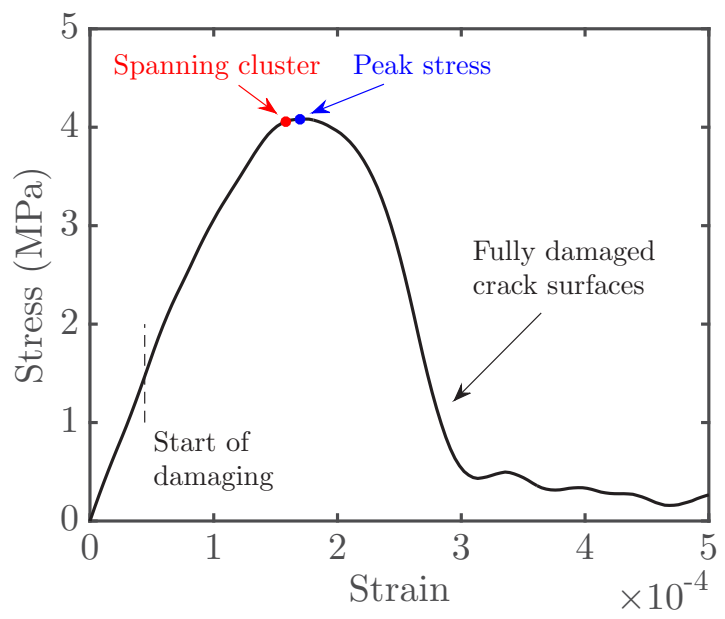

FIG. 2. Mechanical response of the concrete model under uniaxial tensile loading for a strain rate $\dot{\varepsilon}=1 \mathrm{~s}^{-1}$ ( $L=100 \mathrm{~mm}$, packing density of $30 \%$, reference case). 

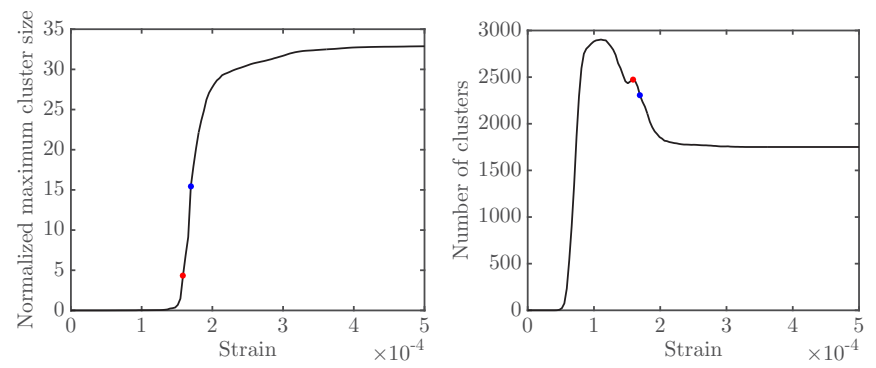

FIG. 3. Maximum cluster size normalized by the cross-sectional area (left) and number of independent clusters (right) as a function of strain for a strain rate $\dot{\varepsilon}=1 \mathrm{~s}^{-1}$ ( $L=100 \mathrm{~mm}$, packing density of $30 \%$, reference case).

into the system. After this, the slope of the stress-strain curve gradually decreases until the peak stress is achieved. At some point along the stress-strain evolution we observe a cluster of damaged elements that spans the entire simulation cell. This is referred to as a system-spanning cluster and is found to occur just prior to the peak stress. Fully damaged crack surfaces are formed only during the postpeak phase causing a sharp drop of the average stress. The tail in the end of the stress-strain curve is due to a bridging of material elements between overlapping crack tips [32].

The evolution of the maximum damage cluster area and the number of the independent clusters are plotted in Fig. 3. The cluster spanning and peak stress points are illustrated with red and blue dots, as in the previous figure. A steep increase for the maximum cluster size is observed in the region of span and peak points. We observe a sharp increase in the number of damage clusters at the onset of damaging. This is because at every aggregate, ITZs are activated at the top and bottom spherical segments perpendicular to the loading direction. Therefore, the maximum number of independent clusters is around twice the aggregate count in the sample. Then the clusters extend to mortar elements and merge with one another thereby decreasing the number of total clusters. The local increase in cluster number at percolation [Fig. 3(b)] is due to the average stress reaching $4 \mathrm{MPa}$, which is the critical stress at which isolated cohesive elements within the mortar can be activated. Both the number of clusters and the maximum cluster area stabilizes after some point as damage develops within the cohesive elements. Although the models are of different nature, an analysis using fiber bundle models showed that the statistics of successive broken fiber patches signal a similar nonmonotonic behavior [33].

We introduce a parameter to measure how far the process is from the system-spanning cluster point as a function of strain and call it the control parameter:

$$
\Delta(\varepsilon)=\frac{\varepsilon_{s}-\varepsilon}{\varepsilon_{s}},
$$

where $\varepsilon_{s}$ is the strain when the system-spanning cluster occurs. $\Delta(\varepsilon=0)=1$ denotes the start of the loading. As the system is loaded, $\Delta$ decreases, eventually becoming zero at the systemspanning cluster. To investigate the statistics of the cluster sizes as a function of the control parameter, we determine the complementary cumulative distribution function, $1-C(S, \Delta)$,
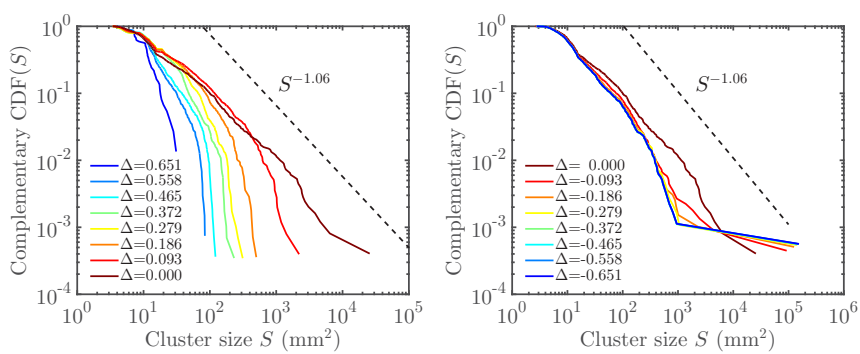

FIG. 4. Complementary CDFs of cluster size before (left) and after (right) percolation for strain rate $\dot{\varepsilon}=1 \mathrm{~s}^{-1}(L=100 \mathrm{~mm}$ with the packing density of $30 \%$, reference case). The distribution converges to an approximate power law as the system approaches percolation (control parameter $\Delta=0$ ). The obtained exponent is the maximum likelihood estimate derived from the data. The right panel demonstrates that the power-law distribution is lost after percolation $(\Delta<0)$

where $C(S, \Delta)$ is the cumulative distribution function (CDF), which gives the probability of observing a cluster size less than $S$ at the control parameter $\Delta$. The visual form of the CDF, $C(S)$, is generally more robust than of the probability distribution function (PDF) against fluctuations due to the finite sample sizes, particularly in the tail of the distribution [34]. The complementary CDFs of the cluster size are illustrated for several $\Delta$, before, at, and after the occurrence of a system-spanning cluster $(\Delta=0)$ in Fig. 4. Inspection of this log-log plot reveals that as $\Delta \rightarrow 0^{+}, 1-C(S, \Delta)$ becomes increasingly powerlaw-like. Whereas for negative values of $\Delta$, after the systemspanning event is seen, there is an increased tendency for larger (system-spanning) clusters to exist, thus destroying the dominant power-law behavior around $\Delta=0$. The damage level [Eq. (10)] of the cohesive elements in the regime of systemspanning clusters is quite low. Hence, at this stage, no macroscopic damage is observed in the sample. Free crack surfaces are formed later along with a drop in the macroscopic stress. The maximum damage observed at this stage is around $6 \%$. We observe that a vast majority of cohesive elements present at percolation contain a little amount of damage $(D<0.01)$.

The onset of a system spanning cluster suggests percolation might be at play. Percolation theory indicates that the PDF of cluster sizes, $S$, should follow a truncated power law [17]:

$$
P(S, \Delta) \sim S^{-\tau} \mathcal{F}\left(S / S_{\xi}\right),
$$

where $\tau$ is the (critical) power-law exponent, $\mathcal{F}$ is a scaling function, and $S_{\xi}$ is the characteristic cluster size which depends on the control parameter $\Delta$. The complementary CDF is then calculated as

$$
1-C(S, \Delta)=\int_{S}^{\infty} P\left(S^{\prime}, \Delta\right) d S^{\prime} \sim S^{-\tau+1} \mathcal{G}\left(S / S_{\xi}\right)
$$

where $\mathcal{G}$ is an another scaling function. Thus, $1-C(S, \Delta)$ also asymptotes to a power-law distribution with critical exponent $(\tau-1)$. The scaling functions, $\mathcal{F}(x)$ and $\mathcal{G}(x)$, both limit to a constant as $x \rightarrow \infty$.

For a bulk (infinite) system, $S_{\xi}$ diverges at $\Delta=0$, resulting in pure power laws for Eqs. (13) and (14). Percolation theory 


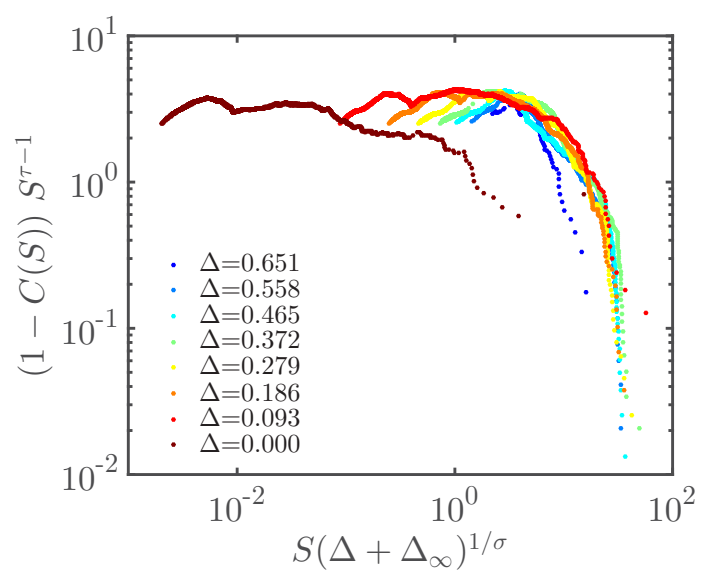

FIG. 5. Scaling collapse of the damage cluster size distributions for the reference system (data given in Fig. 4 for several $\Delta$ values) with $\tau=1.75, \sigma=0.62$, and $\Delta_{\infty}=0.01$.

has $S_{\xi}$ diverging as a power law in terms of $\Delta$ :

$$
S_{\xi} \propto \Delta^{-1 / \sigma} \text { for } \Delta \rightarrow 0
$$

where $\sigma$ is another critical exponent. For a finite system, percolation corresponds to a system-spanning event and an $S_{\xi}$ which is finite, resulting in a truncated power law even at percolation. Thus, for a finite system, Eq. (15) must be modified to $S_{\xi} \propto\left(\Delta+\Delta_{\infty}\right)^{-1 / \sigma}$, where $\Delta_{\infty}$ becomes the control parameter value for the bulk system at $\Delta=0$, when the first system-spanning cluster is observed for a finite system.

The applicability of Eq. (14) to the current problem may be seen by plotting $[1-C(S, \Delta)] S^{\tau-1}$ versus $S \Delta^{1 / \sigma}$ for different values of $\Delta$ to obtain the scaling function $\mathcal{G}(x)$. Figure 5 does this, displaying a reasonable collapse for the values of $\tau=1.75, \sigma=0.62$, and $\Delta_{\infty}=0.01$ over a range spanning two orders of magnitude. The poorest collapse is seen for the $\Delta=0$ case. This is because the distribution at the system-spanning event is predominantly a power law. Because of this, we now consider in detail the measurement of the exponent at percolation.

The power-law fit to the cluster size data at percolation has a lower bound, denoted by $S_{\min }$, meaning that the fit is valid only for $S \geqslant S_{\min }$. The exponent, $\tau$, and $S_{\min }$ are estimated using the method of maximum likelihood. Then, the goodness-of-fit between the data and the power law is calculated using synthetic data sets generated using the estimated $\tau$ and $S_{\min }$. Each synthetic data set is fitted to its own power law, and the Kolmogorov-Smirnov (KS) statistic, a common measure to quantify the distance between two probability distributions, is calculated [35]. The KS statistic is simply the maximum distance between the CDFs of the data and the fitted model. Then the fraction of synthetic realizations for which the resulting statistic is larger than the value for the empirical data is counted. This fraction is called the $p$ value. If $p$ is close to 1 , then the difference between the empirical data and the model can be attributed to statistical fluctuations alone. If it is small, the model is not a plausible fit for the data. The rule of thumb used to reject a power-law fit is $p \leqslant 0.05$. The uncertainties of our estimates $\tau$ and $S_{\min }$ are also calculated via the synthetic data sets. A nonparametric bootstrap method is used, the synthetic data is generated by drawing a new sequence of points uniformly, at random, from the original data with replacement. Using the same fitting methodology, we estimate $\tau$ and $S_{\min }$ for this surrogate data set. The standard deviation of these estimates over a large number of repetitions gives an estimation of the uncertainty in the original estimated parameters. We present the uncertainties in parenthesis next to the estimated variables. For further details regarding to the fitting and testing processes, see Ref. [34].

For the reference sample, the distribution of cluster size converges to a power law with an exponent $\tau=2.06(0.09)$ as we reach the point, when a spanning cluster is first observed $(\Delta=0)$. The power-law distribution is destroyed after percolation due to the coalescence of smaller clusters with the percolated cluster. The size distribution of damage clusters has previously been studied using quite different models. A power-law distribution of crack sizes was also observed with a scalar spring model, and it was reported that the distribution was exponential for the low-strain loadings and approaches to a power law as the loading increases [36]. Girard et al. [12] saw an identical trend of the damage cluster size distribution for their finite-element model, applying a random distribution of disorder. In their case, the size distribution of the damage clusters follows a power law with an exponential cutoff at the peak load under compressive loading. The power-law distribution of cluster sizes found with the models listed above is not in agreement with the lognormal distributions observed in the random fuse models [4].

Power-law distributions of rupture events show variability in terms of the exponent depending on the heterogeneity, stress, brittle or ductile transition, finite size effects, and proximity to the failure [37]. The exponent obtained for the fiber bundle models is 1.86 [38], while scalar spring models yield $\tau=$ 2 [36]. Progressive damage finite-element models under the compressive loading give different exponents with different levels of heterogeneity as the reported values range from 2.6 to 3.6 [12]. It is known that the percolation theory calculates the Fisher's exponent in two dimensions as $\tau_{2 \mathrm{D}}=187 / 91 \approx 2.05$ and $\tau_{3 \mathrm{D}}=2.18$ in three dimensions [17]. The exponent we calculate for the reference case $\tau=2.06 \pm 0.09$ is slightly lower than the prediction of the theory for three dimensions. This shows that larger damage zones occur more frequently due to the correlated growth mechanism and coalescence of clusters as in [38]. However, special attention should be paid to the difference in $\tau$ values obtained by the scaling collapse analysis and a power-law fit at $\Delta=0$. This disparity shows the influence of finite size effects in the reference system.

In what follows, we now consider the effect of different mesostructures and inertial effects by varying the mesostructure properties and the applied strain rate.

\section{A. Random spatial distribution of aggregates}

Ten mesostructures, which have the same mesostructural properties of the one in Fig. 1(d) are analyzed to examine the influence of the spatial arrangement of the aggregates. The samples have the same aggregate size distribution but differ in terms of the placement as this is a random process. The macroscopic stress and cluster evolution do not vary significantly, and the conclusions drawn from the reference 

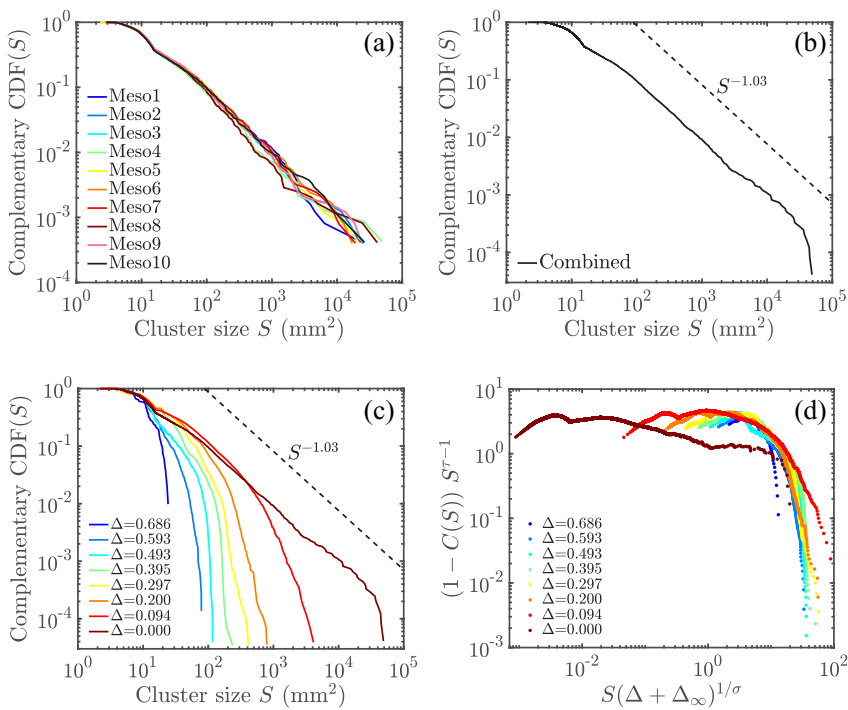

FIG. 6. Complementary CDFs of cluster size at percolation for 10 different mesostructures with $L=100 \mathrm{~mm}$, packing density of $30 \%$, and $\dot{\varepsilon}=1 \mathrm{~s}^{-1}$ (a) and the averaged complementary CDF (b). Fitted exponents are listed in Table II. The combined exponent is $\tau=2.03(0.02)$ with the uncertainty given in parentheses. Combined damage cluster size distributions for several $\Delta$ is given in (c) and its data collapse with $\tau=1.77, \sigma=0.59$, and $\Delta_{\infty}=0.01$ is in (d).

case apply to all of them. Combining several realizations with the same properties removes some of the fluctuations as shown in Fig. 6, while exponents similar to the reference case are found for the data collapse of the combined damage cluster size distributions. Truncation of clusters that are comparable

TABLE II. Estimated $\tau$ and $S_{\min }$ for several (10 for $\dot{\varepsilon}=1 \mathrm{~s}^{-1}$ and three for $\dot{\varepsilon}=10 \mathrm{~s}^{-1}$ ) realizations of aggregate placement (packing density of $30 \%$ ). $p$ value shows the plausibility of the proposed power-law fit.

\begin{tabular}{lccc}
\hline \hline & & $\dot{\varepsilon}=1 \mathrm{~s}^{-1}$ & \\
\cline { 2 - 4 } & $\tau$ & $S_{\min }\left(\mathrm{mm}^{2}\right)$ & $p$ value \\
\hline Meso1 & $2.06(0.09)$ & $80.6(41.4)$ & 0.283 \\
Meso2 & $2.03(0.09)$ & $77.6(39.9)$ & 0.663 \\
Meso3 & $2.03(0.09)$ & $75.9(39.5)$ & 0.542 \\
Meso4 & $2.01(0.07)$ & $46.4(14.2)$ & 0.425 \\
Meso5 & $2.06(0.09)$ & $76.5(40.6)$ & 0.429 \\
Meso6 & $2.00(0.07)$ & $74.8(23.4)$ & 0.649 \\
Meso7 & $1.98(0.07)$ & $64.1(22.5)$ & 0.724 \\
Meso8 & $2.09(0.13)$ & $129.3(38.9)$ & 0.307 \\
Meso9 & $2.04(0.07)$ & $58.6(21.6)$ & 0.950 \\
Meso10 & $2.00(0.10)$ & $70.7(39.8)$ & 0.800 \\
Combined & $2.03(0.02)$ & $79.7(27.0)$ & 0.512 \\
& & $\dot{\varepsilon}=10 \mathrm{~s}^{-1}$ & \\
\cline { 2 - 4 } & & $S_{\min }\left(\mathrm{mm}^{2}\right)$ & $p$ value \\
\hline Meso1 & $1.97(0.10)$ & $92.8(33.2)$ & 0.038 \\
Meso2 & $1.99(0.13)$ & $90.4(48.5)$ & 0.670 \\
Meso3 & $1.92(0.15)$ & $66.3(83.6)$ & 0.038 \\
Combined & $1.97(0.06)$ & $95.7(47.5)$ & 0.135 \\
\hline \hline
\end{tabular}

TABLE III. Random distribution of the tensile strength of the ITZ and mortar paste. Levels from 0 to 3 are introduced to account for different ranges of uniform distribution. Fracture energy is kept constant for all cases.

\begin{tabular}{lcc}
\hline \hline Level & $f_{\text {ct,ITZ }}(\mathrm{MPa})$ & $f_{\text {ct, mor }}(\mathrm{MPa})$ \\
\hline L0 (constant) & 2.4 & 4 \\
L1 & $1.4-3.4$ & 4 \\
L2 & $0.4-4.4$ & 4 \\
L3 & $0.4-4.4$ & $2-6$ \\
\hline \hline
\end{tabular}

to the system size at $\Delta=0$ is also observed with the combined set of data.

Cluster size distributions of the mesostructures are consistent within their range of uncertainty. The exponent of the combined distribution $\tau=2.03(0.02)$ was obtained by summing the clusters of all mesostructures at percolation. Table II lists the power-law estimates for strain rates of 1 $\mathrm{s}^{-1}$ and $10 \mathrm{~s}^{-1}$. The uncertainty for the exponent is reduced with combining cluster size data. For the $10 \mathrm{~s}^{-1}$ case, first and third mesostructure have $p$ values lower than 0.05 , but combined data give a sufficient $p$ value to accept the power-law distribution hypothesis.

\section{B. Random material parameters}

Up to this point, the only heterogeneity present in the model is from the mesostructure itself and the weak zones of the ITZ are always the primary damage initiation locations. We introduce an additional degree of uncertainty by introducing a uniform distribution to the tensile strength of ITZ and
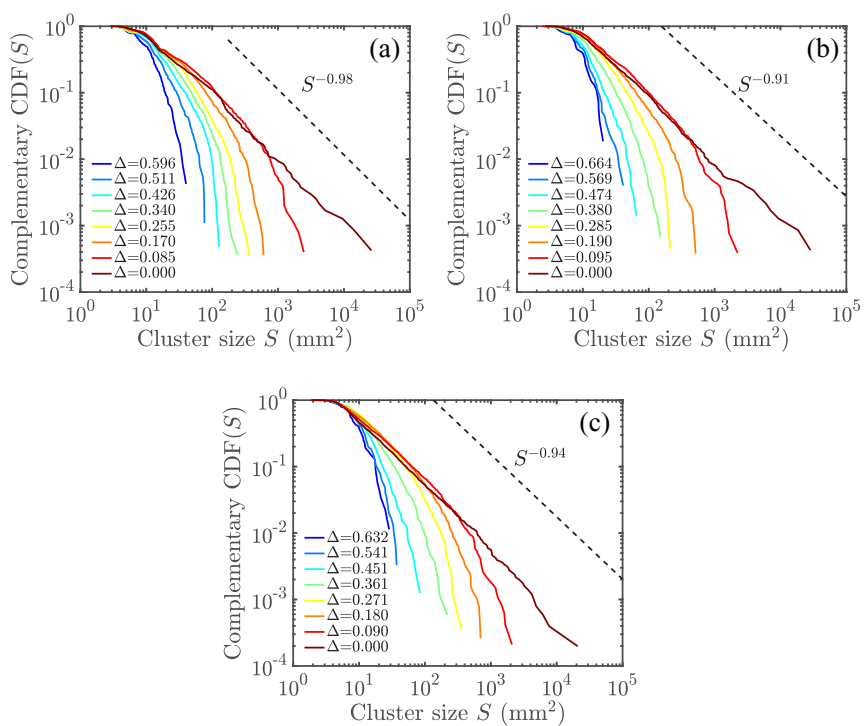

FIG. 7. Complementary CDFs of cluster sizes for several material randomness levels ( $L=100 \mathrm{~mm}$, packing density of $30 \%, \dot{\varepsilon}=1 \mathrm{~s}^{-1}$, reference case). $L 1$ (a), $L 2$ (b), and $L 3$ (c) plots show that in all cases cluster distribution converge to a power law at percolation. The power-law fit is valid for smaller clusters, and the fluctuations reduce as the material randomness increases, see the estimates of $S_{\min }$ in Table IV. 
TABLE IV. Estimated $\tau$ and $S_{\min }$ for four distribution levels ( $L=$ $100 \mathrm{~mm}$, packing density of $30 \%, \dot{\varepsilon}=1 \mathrm{~s}^{-1}$, reference case). $p$ value shows the plausibility of the proposed power-law fit.

\begin{tabular}{lccc}
\hline \hline Level & $\tau$ & $S_{\min }\left(\mathrm{mm}^{2}\right)$ & $p$ value \\
\hline$L 0$ & $2.06(0.09)$ & $80.6(41.4)$ & 0.283 \\
$L 1$ & $1.98(0.06)$ & $173.8(30.0)$ & 0.745 \\
$L 2$ & $1.91(0.04)$ & $11.2(7.7)$ & 0.392 \\
$L 3$ & $1.94(0.03)$ & $5.9(6.0)$ & 0.063 \\
\hline \hline
\end{tabular}

mortar paste. The standard deviation is gradually increased (Table III) within a range of material properties observed experimentally [39].

$L 0$ is the base level (the reference case). Complementary CDFs for all levels are presented in Fig. 7.

In all cases, the size distribution of the clusters follows a power law at percolation. A summary of the power-law exponent estimates is given in Table IV. $S_{\min }$ values for levels $L 2$ and $L 3$ show the fit is valid for a larger range of cluster sizes as we increase material randomness. In addition, fluctuations in the distribution curve at percolation reduce as the decrease in the fit uncertainty shows. The exponents for $L 1, L 2$, and $L 3$ are all lower than the constant material parameter case, $L 0$.

A fair scaling collapse, including the data set at $\Delta=0$ is obtained with the addition of random material parameters in Fig. 8. Furthermore, the Fisher's exponent derived from the scaling collapse analysis is very close to the one obtained from the power-law fit at $\Delta=0$ unlike the reference case, in which we use constant material parameters. Thus, injecting material randomness to the reference case, tends to give better statistics, and therefore improved mesostructure self-averaging for this considered sample size.

It should be noted that under extreme disorder, crack cluster properties were found to differ from the random percolation statistics using a random fuse network model [40]. The present work shows that when the disorder is within a range of experimentally observed material properties, random

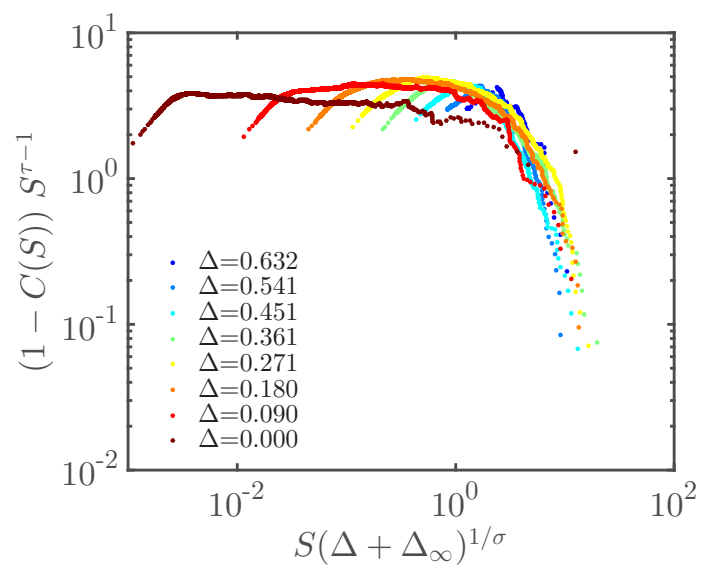

FIG. 8. Scaling collapse of the damage cluster size distributions of the level $L 3$ (data given in Fig. 7c for several $\Delta$ values) with with $\tau=1.90, \sigma=0.34$, and $\Delta_{\infty}=0.08$.
TABLE V. Estimated $\tau$ and $S_{\text {min }}$ for several packing densities (PD) ( $L=100 \mathrm{~mm}, \dot{\varepsilon}=1 \mathrm{~s}^{-1}$ ). $p$ value shows the plausibility of the proposed power-law fit. $\eta$ for each mesostructure is given in brackets.

\begin{tabular}{lccc}
\hline \hline $\operatorname{PD}[\eta]$ & $\tau$ & $S_{\min }\left(\mathrm{mm}^{2}\right)$ & $p$ value \\
\hline $20 \%[0.2]$ & $1.91(0.04)$ & $15.0(12.6)$ & 0.260 \\
$30 \%[0.1]$ & $2.06(0.09)$ & $80.6(41.4)$ & 0.283 \\
$40 \%[0.04]$ & $2.12(0.11)$ & $84.3(31.4)$ & 0.744 \\
\hline \hline
\end{tabular}

percolation statistics prevails, indicating a weak disorder regime for the current model.

\section{Packing density}

To study the effect of packing density, mesostructures containing $20 \%, 30 \%$, and $40 \%$ of coarse aggregates are constructed using the take-and-place method with sufficiently high $\eta$ values for each case to obtain a homogeneous distribution of aggregates; see Table V. A strain rate of $\dot{\varepsilon}=1 \mathrm{~s}^{-1}$ is applied to all samples. The mechanical response and the number of independent clusters are compared in Fig. 9.

Coarse aggregates have larger elastic modulus than mortar paste, therefore the linear elastic response is stiffer for larger packing densities. Also, more aggregates mean more weak regions of ITZ, hence the samples with higher aggregate content yield more damaged regions during loading. For this reason lower packing densities attain higher peak stresses. A spanning cluster is observed just before peak stress in all cases.

There are differences in the evolution of the number of independent clusters. For all three cases, the number of clusters increases first around two times the number of the aggregates as the ITZs are activated at the top and bottom spherical segments perpendicular to the loading direction. The local increase we observed before for the sample of $30 \%$ aggregate becomes an influential peak for the $20 \%$ case. The reason lies in the constitutive response. Since the tensile strength of the mortar-mortar interface is $4 \mathrm{MPa}$, and the $20 \%$ case attains stresses higher than this value before the peak, there are cohesive elements activated in the mortar paste for damage initiation. For the same reason, we have no further increase of independent clusters after the ITZs of the aggregates are activated for the $40 \%$ case as the average stress never exceeds $4 \mathrm{MPa}$.
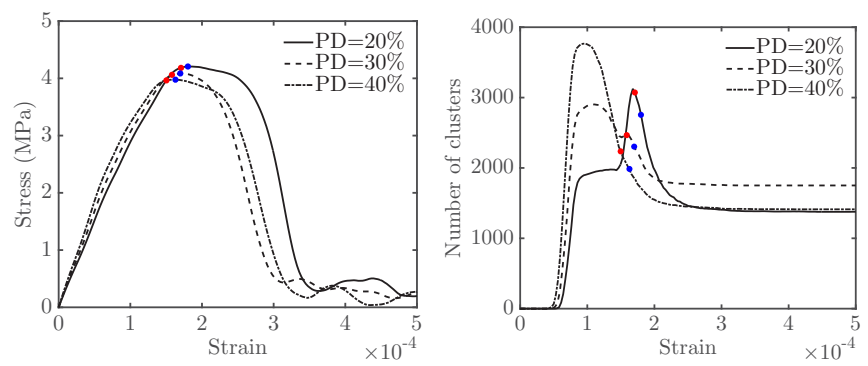

FIG. 9. Mechanical response (left) and number of independent clusters (right) for the mesostructures with packing densities (PD) of $20 \%, 30 \%$, and $40 \%$. Red circles denote the cluster-spanning point while blue circles are for the peak stress $\left(L=100 \mathrm{~mm}, \dot{\varepsilon}=1 \mathrm{~s}^{-1}\right)$. 

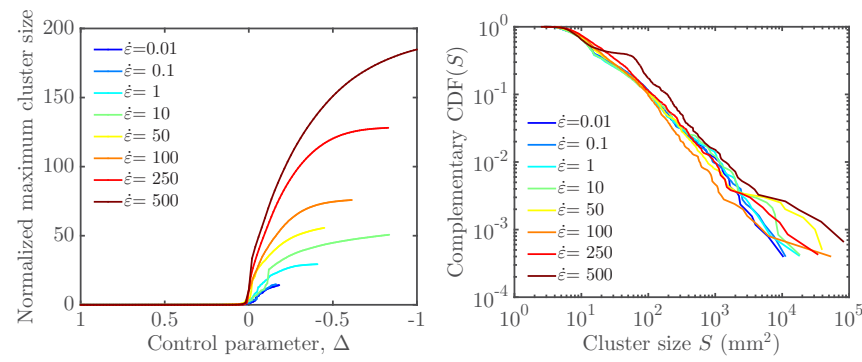

FIG. 10. Normalized maximum cluster size evolution (left) and complementary CDFs (right) at percolation for various strain rates $\left(\dot{\varepsilon}=0.01-500 \mathrm{~s}^{-1}\right)$. All curves are obtained using the reference mesostructure ( $L=100 \mathrm{~mm}$, packing density of $30 \%)$.

The complementary CDFs of $20 \%$ and $40 \%$ cases (not given in the manuscript) behave in an identical manner as the $30 \%$ curves. The size distribution converges to a power law at percolation, and the power law is lost upon further loading. Estimated power-law fit variables are listed in Table V. We observe higher exponents with increased heterogeneity.

\section{Strain rate}

The strain rate is altered to investigate how inertia affects the field-induced percolation analogy. We consider a range of strain rates spanning $\dot{\varepsilon}=0.01-500 \mathrm{~s}^{-1}$. The maximum cluster size evolution and complementary CDFs at percolation are plotted in Fig. 10.

Larger clusters are achieved with faster rates as they allow more diffuse damaging in the concrete sample. Diffuse damage zones coalesce and form larger clusters during loading. The size distributions are consistent with increasing fluctuation at higher rates. Estimated power-law fit parameters are given in Table VI. We see that at high rates $\dot{\varepsilon} \geqslant 10 \mathrm{~s}^{-1}$, there is more noise in the data. However, we do not observe a systematic dependence of the $\tau$ exponent on the strain rate. Accordingly, low $p$ values are observed in the high rate regime. However, for all rates considered, the size distribution converges to a power law at percolation, and afterwards, it is destroyed with an overgrowing dominant cluster.

TABLE VI. Estimated $\tau$ and $S_{\min }$ for several strain rates ( $L=$ $100 \mathrm{~mm}$, packing density of $30 \%$ ). $p$ value shows the plausibility of the proposed power-law fit.

\begin{tabular}{lccc}
\hline \hline$\dot{\varepsilon}\left(\mathrm{s}^{-1}\right)$ & $\tau$ & $S_{\min }\left(\mathrm{mm}^{2}\right)$ & $p$ value \\
\hline 0.01 & $2.11(0.07)$ & $75.9(14.6)$ & 0.603 \\
0.1 & $2.11(0.09)$ & $79.2(26.0)$ & 0.192 \\
1 & $2.06(0.09)$ & $80.6(41.4)$ & 0.283 \\
10 & $1.97(0.10)$ & $92.8(33.2)$ & 0.038 \\
50 & $1.82(0.10)$ & $8.7(20.9)$ & 0.000 \\
100 & $2.30(0.22)$ & $86.2(38.8)$ & 0.484 \\
250 & $2.02(0.05)$ & $42.0(17.8)$ & 0.199 \\
500 & $1.94(0.10)$ & $357.7(75.1)$ & 0.673 \\
\hline \hline
\end{tabular}
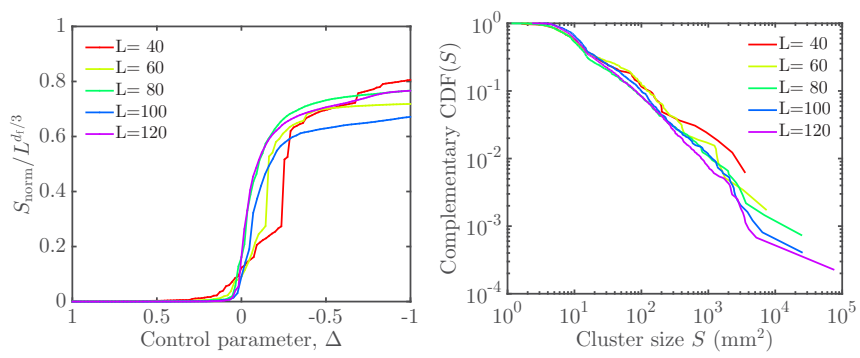

FIG. 11. Normalized maximum cluster size $\left(S_{\text {norm }}\right)$ evolution (left) and complementary CDFs (right) at percolation for various sample sizes $(L=40-120 \mathrm{~mm})$. The mesostructures presented in Fig. 1 are used for the analyses with strain rate $\dot{\varepsilon}=1 \mathrm{~s}^{-1}$.

\section{E. Simulation box size}

The sample sizes of $L=40,60,80,100$, and $120 \mathrm{~mm}$ are considered to examine the size effect; see Fig. 1. The maximum cluster size evolution and complementary CDFs at percolation are plotted in Fig. 11 for the strain rate $\dot{\varepsilon}=1 \mathrm{~s}^{-1}$. The vertical axis of Fig. 11(a) is plotted as the normalized maximum cluster size divided by $L^{d_{\mathrm{f}} / 3}$ where $d_{\mathrm{f}}$ is the fractal dimension at percolation. Here the three-dimensional percolation value of $d_{\mathrm{f}}=2.52$ is used. The good collapse of the vertical scale with respect to this scaling gives further support of an underlying percolation phenomenon.

The fractal dimensions of the spanning clusters may also be estimated for different sample sizes using a Hausdorff boxcounting algorithm and are listed in Table VII. The estimation increases with size but is always lower than the theoretical value of $d_{\mathrm{f}}=2.52$, demonstrating the limitation of our finite sample size.

The distributions again approach a power law as the system is driven to percolation. Estimated power-law fit parameters are listed in Table VIII for strain rates $1 \mathrm{~s}^{-1}$ and $10 \mathrm{~s}^{-1}$. It is observed that the exponent for $L=40 \mathrm{~mm}$ case is lower compared to others. It must be noted that this size cannot be considered as a representative volume as the maximum aggregate diameter is $16 \mathrm{~mm}$, nearly half the size of the simulation box size. Hence, the clusters are severely influenced by the spatial arrangement of comparatively big aggregates. Additionally, the range of the power law reduces as simulationbox size decreases.

As in Figs. 4 and 6, Fig. 11(b) demonstrates a distribution at $\Delta=0$ which is predominantly a power law. This appears not to change with system size, which is somewhat surprising given that the smaller the system, the further the $\Delta=0$ state is from the true bulk critical point.

TABLE VII. Fractal dimension estimations for different simulation-box sizes with the uncertainties given in parentheses.

\begin{tabular}{lc}
\hline \hline$L(\mathrm{~mm})$ & Fractal dimension, $d_{\mathrm{f}}$ \\
\hline 60 & $2.06(0.12)$ \\
80 & $2.17(0.12)$ \\
100 & $2.18(0.12)$ \\
120 & $2.27(0.15)$ \\
\hline
\end{tabular}


TABLE VIII. Estimated $\tau$ and $S_{\min }$ for several sample sizes (packing density of 30\%). $p$ value shows the plausibility of the proposed power-law fit.

\begin{tabular}{|c|c|c|c|c|c|c|}
\hline \multirow[b]{2}{*}{$L(\mathrm{~mm})$} & \multicolumn{3}{|c|}{$\dot{\varepsilon}=1 \mathrm{~s}^{-1}$} & \multicolumn{3}{|c|}{$\dot{\varepsilon}=10 \mathrm{~s}^{-1}$} \\
\hline & $\tau$ & $S_{\min }\left(\mathrm{mm}^{2}\right)$ & $p$ value & $\tau$ & $S_{\min }\left(\mathrm{mm}^{2}\right)$ & $p$ value \\
\hline 40 & $1.70(0.09)$ & $6.2(9.0)$ & 0.136 & $1.71(0.13)$ & $8.5(14.1)$ & 0.262 \\
\hline 60 & $2.01(0.21)$ & $63.5(31.3)$ & 0.932 & $1.93(0.13)$ & $50.1(22.0)$ & 0.878 \\
\hline 80 & $1.91(0.09)$ & $57.6(25.1)$ & 0.914 & $1.80(0.08)$ & $14.7(23.1)$ & 0.025 \\
\hline 100 & $2.06(0.09)$ & $80.6(41.4)$ & 0.283 & $1.97(0.10)$ & $92.8(33.2)$ & 0.038 \\
\hline 120 & $2.08(0.11)$ & $137.3(49.4)$ & 0.652 & $2.00(0.07)$ & $70.9(22.8)$ & 0.659 \\
\hline
\end{tabular}

It has been shown that in the limit of large system sizes, percolation-like behavior vanishes and the failure can be described by nucleation; see the phase diagram in Ref. [41]. Our samples suffer from a strong finite-size effect and are not near such a limit. Since we observe neither nucleation nor scale-free avalanches, it is natural that our simulations should fall in the percolation regime.

\section{PERCOLATED DAMAGE CLUSTER AND FINAL CRACKING}

Figure 12 visualizes the damage clusters obtained at percolation and the final macroscopic crack surface, for three realizations of the reference mesostructure [Fig. 1(d)] for the strain rate $\dot{\varepsilon}=1 \mathrm{~s}^{-1}$. At this strain rate, the crack is well localized.

It was mentioned before that the average damage in cohesive elements prior to yield is low, with the maximum damage level being around 5\%-6\% at percolation. Cohesive elements with comparatively high damage are diffused randomly in the cluster at this level of the analysis, so the damage map itself does not give great insight for the final crack profile. For two samples, Fig. 12 shows that the location of the percolated cluster is qualitatively close to the final cracking. However, in one of the mesostructures (central in Fig. 12), a large part of the final crack is formed far from the percolated damage cluster

The percolated cluster and final cracking are now quantitatively compared in terms of the cohesive elements they contain; see Table IX. For the strain rate $1 \mathrm{~s}^{-1}$, it is observed that only $11 \%$ of the spanning cluster becomes fully damaged in the end, while one-third of the final crack is formed by the elements belonging to the percolated cluster. It is also interesting that on average around $44 \%$ of the free surface was intact at percolation. This shows that the cracking of the sample at the postpeak phase is due to the further initiation and nucleation of damage zones. The little-damaged percolated cluster is therefore uncorrelated with the final macroscopic crack. Random fuse models also show that the largest crack clusters, at the peak load, do not propagate into a spanning crack, and the final crack is formed due to the coalescence of smaller cracks [4].

Since the percolated cluster is mostly constituted of lowdamage elements, one meaningful question is whether the elements that have comparatively high damage in these clusters act as weak spots and are responsible for the free surfaces upon the failure. To investigate this aspect, we set a damage threshold for all of the elements at percolation and monitor the percentage of those elements above this threshold, and belonging to the percolating cluster, becoming free surfaces later; see Fig. 13.
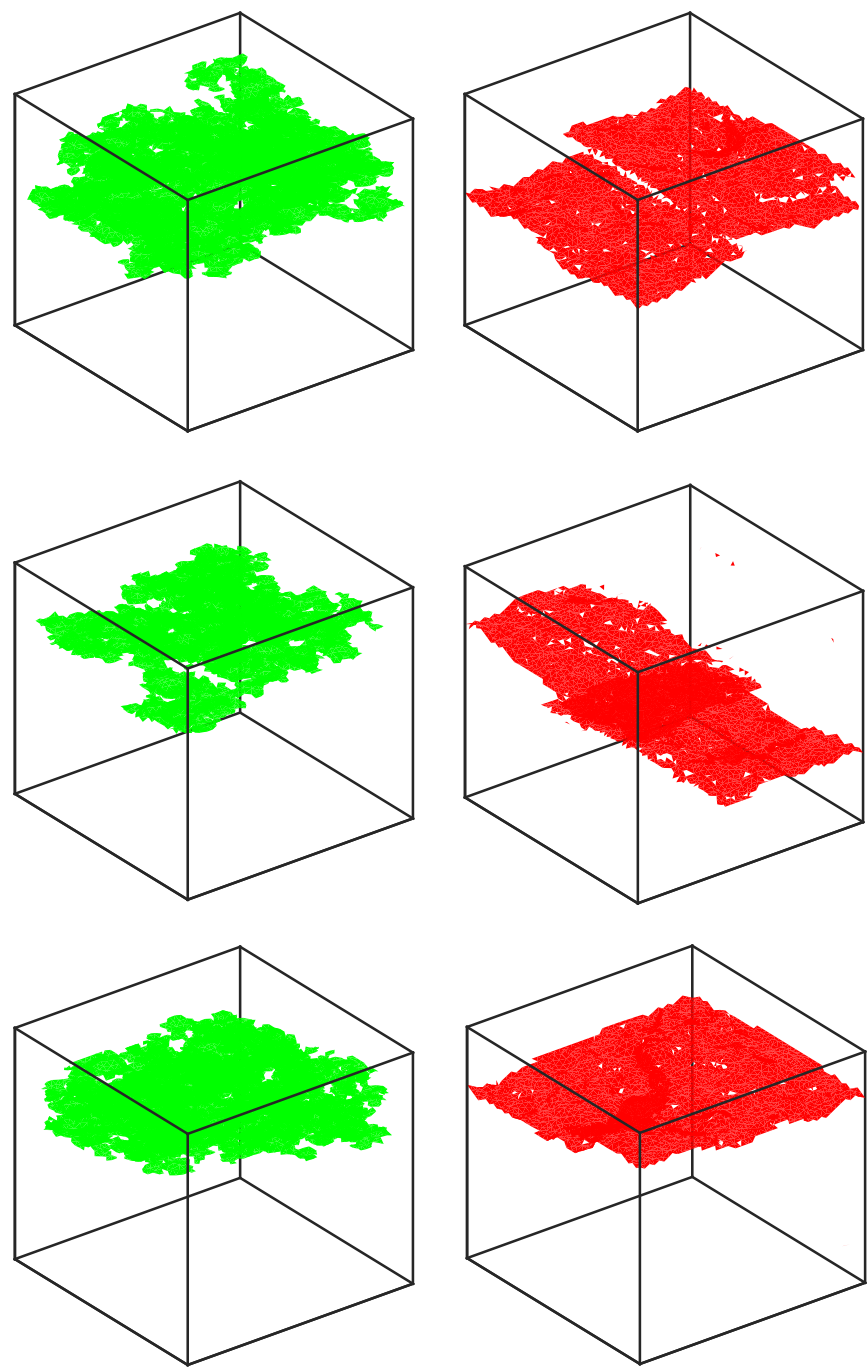

FIG. 12. Percolated clusters (left) and the free surfaces formed upon the failure (right) for three different realizations of the mesostructure in Fig. 1(d) for a strain rate $\dot{\varepsilon}=1 \mathrm{~s}^{-1}$. While all cohesive elements with damage $D>0$ are plotted on the left for the percolated clusters, only the totally damaged cohesive elements are shown on the right. 
TABLE IX. Comparison of the percolated cluster (PC) and final cracking (FC). Meso1 to Meso10 are the 10 different realizations of the mesostructure illustrated in Fig. 1(d) at strain rate $\dot{\varepsilon}=1 \mathrm{~s}^{-1}$. PC/FC: the amount of PC becoming FC, FC/PC: the amount of FC present in $\mathrm{PC}, \mathrm{FC} /$ perc.: the amount of $\mathrm{FC}$ present at percolation. The cases where more than one percolated cluster is observed at percolation are denoted with an asterisk.

\begin{tabular}{lccc}
\hline \hline & PC/FC & FC/PC & FC/perc. \\
\hline Meso1 & $14.80 \%$ & $46.18 \%$ & $57.45 \%$ \\
Meso2 & $11.74 \%$ & $20.27 \%$ & $55.74 \%$ \\
Meso3 & $17.40 \%$ & $47.99 \%$ & $55.72 \%$ \\
Meso4* & $7.37 \%$ & $58.40 \%$ & $62.05 \%$ \\
Meso5* & $9.67 \%$ & $32.09 \%$ & $54.24 \%$ \\
Meso6 & $7.63 \%$ & $7.47 \%$ & $53.69 \%$ \\
Meso7 & $17.77 \%$ & $34.80 \%$ & $49.75 \%$ \\
Meso8* & $8.51 \%$ & $50.97 \%$ & $58.28 \%$ \\
Meso9 & $0.00 \%$ & $0.00 \%$ & $55.84 \%$ \\
Meso10 & $13.17 \%$ & $33.00 \%$ & $58.13 \%$ \\
Average & $10.81 \%$ & $33.12 \%$ & $56.09 \%$ \\
\hline \hline
\end{tabular}

Figure 13 demonstrates that comparatively high-damaged elements at percolation are more likely to become fully damaged upon failure. Considering all the elements present at percolation, in six mesostructures out of 10 , all of the elements having the most damage contribute to the free surfaces. However, in the remaining four, some portion of the most-damaged zones does not get fully damaged when the sample fails. When we limit our inspection to the percolated cluster, its lack of correlation with the final cracking profile becomes more clear. The effect of the weak spots at percolation varies depending on the mesostructure, but, overall, the amount of damage increases the likelihood that the element will be fully damaged. However, in four out of 10 mesostructures the weakest elements in the percolated cluster become a part of the final cracking profile, while in others, they stop carrying further damage. It is interesting to note that in one case, the percolated cluster has no common elements with the final cracking. Hence, the final cracking is not necessarily obtained by further damaging the spanning cluster. Instead, it is a result of a more complex growth and coalescence mechanism, which includes the elements that are inserted after percolation.

Figure 14 displays the percolated cluster and free surfaces upon failure for the higher strain rate $\dot{\varepsilon}=10 \mathrm{~s}^{-1}$. In this higher rate regime, where we lose the crack localization and the clusters at percolation give no hint on where the free surfaces are going to be. As the strain rate increases, the number of cracked cohesive elements increase and several crack clusters of various sizes exist at failure. Figure 15 shows the crack morphologies as a function of strain rate.

Crack localization is clearly lost at higher strain rates with the cracked region occupying the whole simulation box. At lower rates the first crack has time to grow creating neighboring damage zones. In other words the emergence of new cracks and their growth is slow enough for localization to happen. However, at higher rates the emergence rate of new cracks is comparable to the growth rate of existing cracks leading to homogeneous failure. To verify this, the percentages of the damage zones at percolation becoming fully damaged
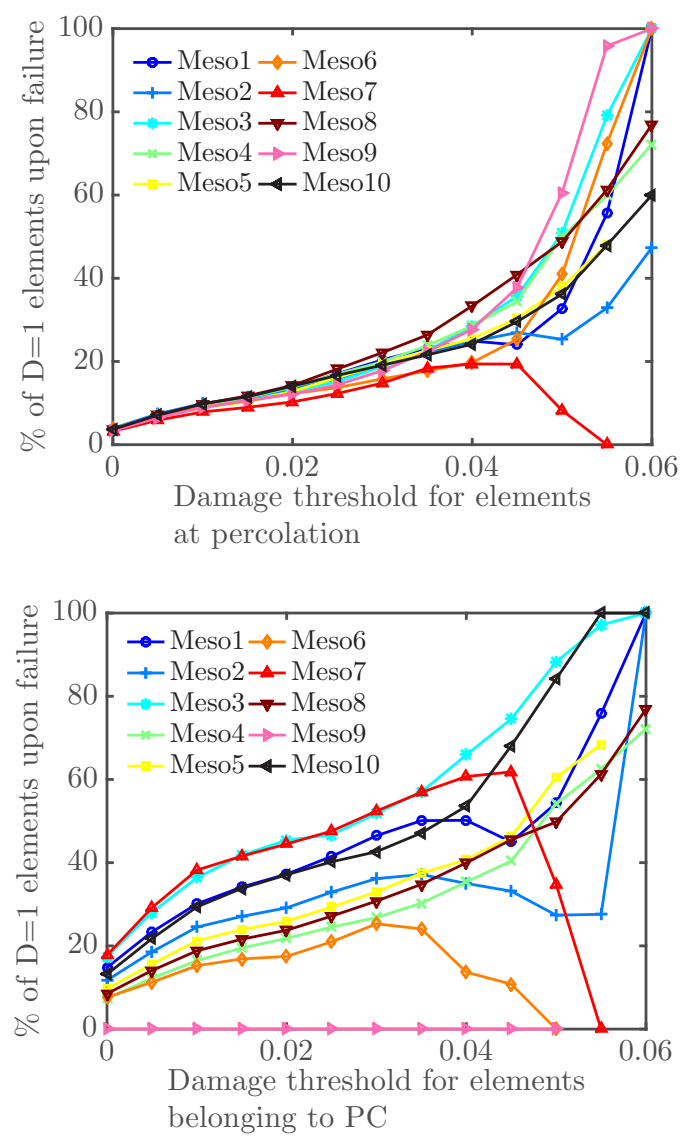

FIG. 13. Percentage of the elements having larger damage from a specified threshold and becoming fully damaged $(D=1)$ upon failure: (above) all elements at percolation and (below) only the elements belonging to the percolated cluster (PC) $(L=100 \mathrm{~mm}$, $\left.\dot{\varepsilon}=1 \mathrm{~s}^{-1}\right)$. The captions Meso1 to Meso10 are the 10 different realizations of the mesostructure illustrated in Fig. 1(d).

crack surfaces are listed in Table $\mathrm{X}$ as a function of strain rate. The likelihood of an existing damage zone to become fully damaged crack surface increases with the strain rate. Furthermore, higher rates activate more damage zones and cause bigger avalanches upon loading. Here an avalanche event is defined as the number of further damaged cohesive elements
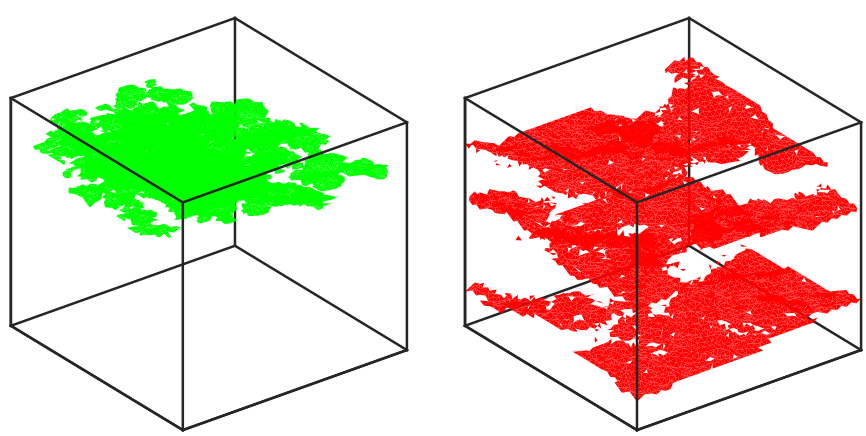

FIG. 14. Percolated cluster (left) and the free surfaces formed upon the failure (right) for strain rate $\dot{\varepsilon}=10 \mathrm{~s}^{-1}$. While all cohesive elements with damage $D>0$ are plotted on the left for the percolated cluster, only the totally damaged cohesive elements are shown on the right. 

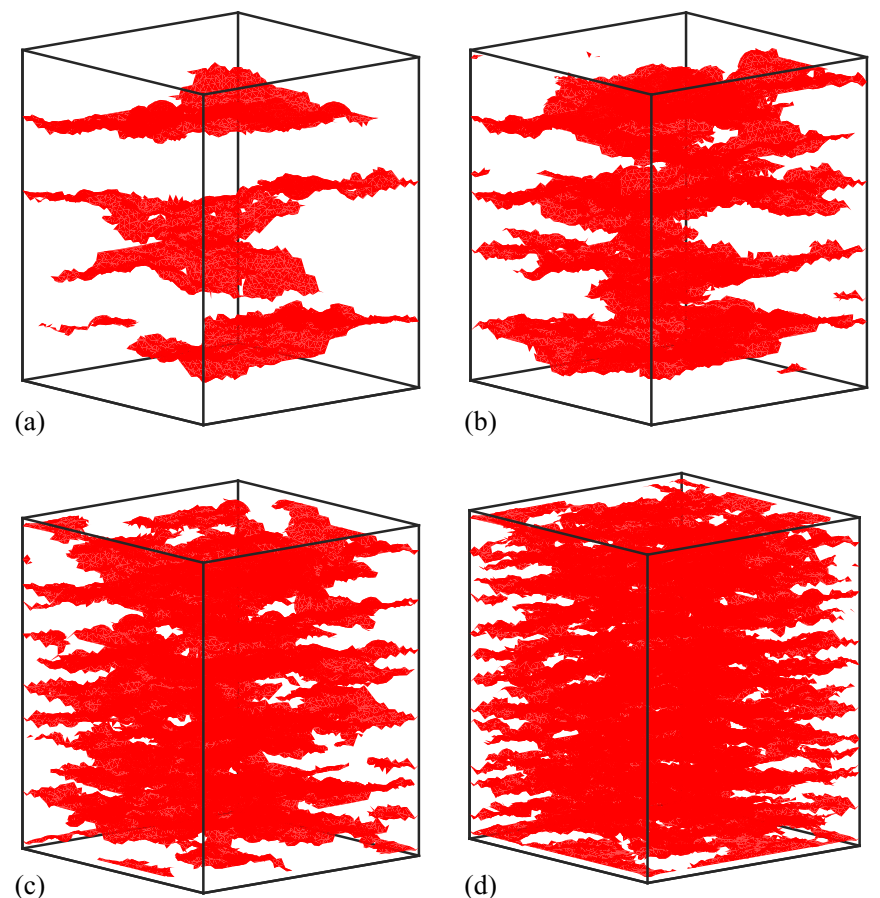

(d)

FIG. 15. Final cracking (fully damaged cohesive elements, $D=1$ ) at high strain rates: (a) $\dot{\varepsilon}=10 \mathrm{~s}^{-1}$, (b) $\dot{\varepsilon}=50 \mathrm{~s}^{-1}$, (c) $\dot{\varepsilon}=100 \mathrm{~s}^{-1}$, (d) $\dot{\varepsilon}=250 \mathrm{~s}^{-1}$.

in a time step. For all cases considered in this study, we observed that avalanches gradually increase as more damage zones are activated with the applied loading and damage increases progressively in cohesive elements with a constant stress threshold. The gradual increase of avalanche size also shows that our system is very different from the random models, for which a plastic plateau is artificially created by continuously reinserting spring elements (or scalar fuses).

\section{CONCLUSIONS}

We studied the statistics of damage clusters in concrete under uniaxial tension at the mesoscale. The size distribution of clusters converges to a power law as the system approaches percolation. The power law is destroyed with further loading. This behavior is insensitive to strain rate, sample size, packing density, and random distribution of aggregates and material parameters within the defined ranges, indicating a universal phenomenon is at play. Distribution exponents are close to the
TABLE X. The amount of damage zones at percolation that forms final cracking (FC) as a function of strain rate.

\begin{tabular}{lc}
\hline \hline$\dot{\varepsilon}\left(\mathrm{s}^{-1}\right)$ & \% of elements at percolation becoming FC \\
\hline 1 & $3.65 \%$ \\
10 & $6.30 \%$ \\
50 & $11.80 \%$ \\
100 & $18.31 \%$ \\
250 & $27.81 \%$ \\
500 & $29.29 \%$ \\
\hline \hline
\end{tabular}

ones found in the literature using random physical models suggesting the phenomenon of a field-induced percolation process [42] as we drive our system to failure by application of strain. Percolation theory suggests $\tau_{3 \mathrm{D}}=2.18$ [17] for three dimensions, and we have smaller exponents, which shows that larger damage zones occur more frequently due to the correlated growth mechanism and coalescence of clusters. More fluctuations are observed for the size distribution as we increase strain rate. The fluctuations reduce when several samples are run and averaged. On the other hand, to obtain a reasonable scaling collapse, a lower cluster size distribution exponent was needed suggesting interaction between the cohesive zone elements might be important.

At lower rates, the damage map at the percolation gives qualitative insights about the emerging free crack surfaces. However, a quantitative analysis at $\dot{\varepsilon}=1 \mathrm{~s}^{-1}$ of cohesive elements show that only a small fraction $(\approx 11 \%)$ of the percolated cluster becomes part of the final crack. In addition, more than $40 \%$ of the cohesive elements that will form the free cracks in the end are not inserted yet at percolation. This verifies the phenomenon observed in random fuse models, that the largest crack clusters at the peak load do not propagate into a spanning crack and the final crack is formed due to the coalescence of smaller cracks. Further analysis shows that the likelihood of an element to be a part of the final cracking increases if the damage level attained at percolation is higher. We observe that the macroscopic crack upon failure does not necessarily emerge from the weak spots of the percolated cluster. Final cracking is a result of a more complex growth and coalescence process, which includes the elements that are inserted after percolation. Hence, the transition to yield (system-spanning damage) is described as a percolation phenomenon while failure (system-spanning crack) is an another mechanical regime with different properties.
[1] H. J. Herrmann and S. Roux, Statistical Models for Fracture in Disordered Media (North-Holland, Amsterdam, 1990).

[2] L. De Arcangelis, S. Redner, and H. J. Herrmann, J. Phys. Lett. 46, 585 (1985).

[3] S. Zapperi, A. Vespignani, and H. E. Stanley, Nature (London) 388, 658 (1997).

[4] S. Nukala, P. Kumar V. V. Nukala, S. Šimunović, and F. Guess, Phys. Rev. E 73, 036109 (2006).

[5] L. de Arcangelis, A. Hansen, H. J. Herrmann, and S. Roux, Phys. Rev. B 40, 877 (1989).
[6] H. J. Herrmann, A. Hansen, and S. Roux, Phys. Rev. B 39, 637 (1989).

[7] F. Peirce, J. Textile Inst. Trans. 17, T355 (1926).

[8] H. Daniels, J. R. Soc. London A 183, 405 (1945).

[9] J. V. Andersen, D. Sornette, and K.-T. Leung, Phys. Rev. Lett. 78, 2140 (1997).

[10] F. Kun, S. Zapperi, and H. J. Herrmann, Eur. Phys. J. B 17, 269 (2000).

[11] K. M. Salerno and M. O. Robbins, Phys. Rev. E 88, 062206 (2013). 
[12] L. Girard, J. Weiss, and D. Amitrano, Phys. Rev. Lett. 108, 225502 (2012).

[13] A. Hansen and S. Roux, in Damage and Fracture of Disordered Materials, edited by D. Krajcinovic and J. G. M. Van Mier (Springer, New York, 2000), pp. 17-101.

[14] M. J. Alava, P. K. V. V. Nukala, and S. Zapperi, Adv. Phys. 55, 349 (2006).

[15] S. Biswas, P. Ray, and B. K. Chakrabarti, Statistical Physics of Fracture, Beakdown, and Earthquake: Effects of Disorder and Heterogeneity (John Wiley \& Sons, New York, 2015).

[16] J. P. Ollivier, J. C. Maso, and B. Bourdette, Advanced Cement Based Materials 2, 30 (1995).

[17] D. Stauffer and A. Aharony, Introduction to Percolation Theory (CRC Press, Boca Raton, FL, 1994).

[18] Z. M. Wang, A. K. H. Kwan, and H. C. Chan, Comput. Struct. 70, 533 (1999).

[19] W. B. Fuller and S. E. Thompson, Trans. Am. Soc. Civ. Eng. 57, 67 (1906).

[20] P. Wriggers and S. O. Moftah, Finite Elements Anal. Design 42 623 (2006).

[21] J. G. M. Van Mier and M. R. A. Van Vliet, Eng. Fract. Mech. 70, 2281 (2003).

[22] T. Belytschko, W. K. Liu, B. Moran, and K. Elkhodary, Nonlinear Finite Elements for Continua and Structures, 2nd ed. (John Wiley \& Sons, New York, 2014).

[23] R. Courant, K. Friedrichs, and H. Lewy, IBM J. Res. Dev. 11, 215 (1967).

[24] F. Gatuingt, L. Snozzi, and J.-F. Molinari, Int. J. Numer. Anal. Methods Geomech. 37, 3112 (2013).

[25] D. S. Dugdale, J. Mech. Phys. Solids 8, 100 (1960).
[26] G. I. Barenblatt, Adv. Appl. Mech. 7, 55 (1962).

[27] G. T. Camacho and M. Ortiz, Int. J. Solids Struct. 33, 2899 (1996).

[28] M. Ortiz and A. Pandolfi, Int. J. Numer. Methods Eng. 44, 1267 (1999).

[29] A. C. Palmer and J. R. Rice, Proc. R. Soc. London A 332, 527 (1973).

[30] A. Hillerborg, M. Modeer, and P. E. Petersson, Cement Concrete Res. 6, 773 (1976).

[31] L. Snozzi, A. Caballero, and J.-F. Molinari, Cement Concrete Res. 41, 1130 (2011).

[32] J. G. M. Van Mier, Cement Concrete Res. 21, 1 (1991).

[33] S. Biswas, S. Roy, and P. Ray, Phys. Rev. E 91, 050105 (2015).

[34] A. Clauset, C. R. Shalizi, and M. E. J. Newman, SIAM Rev. 51, 661 (2009).

[35] F. J. Massey Jr., J. Am. Stat. Assoc. 46, 68 (1951).

[36] C. Spyropoulos, C. H. Scholz, and B. E. Shaw, Phys. Rev. E 65, 056105 (2002).

[37] D. Amitrano, Eur. Phys. J. Special Topics 205, 199 (2012).

[38] K. Kovács, R. C. Hidalgo, I. Pagonabarraga, and F. Kun, Phys. Rev. E 87, 042816 (2013).

[39] C. Rosselló and M. Elices, Cement Concrete Res. 34, 1441 (2004).

[40] A. A. Moreira, C. L. N. Oliveira, A. Hansen, N. A. M. Araújo, H. J. Herrmann, and J. S. Andrade Jr., Phys. Rev. Lett. 109, 255701 (2012).

[41] A. Shekhawat, S. Zapperi, and J. P. Sethna, Phys. Rev. Lett. 110, 185505 (2013).

[42] M. Aertsens and J. Naudts, J. Stat. Phys. 62, 609 (1991). 